\title{
Supercontinuum Generation with femtosecond pulse fiber amplified VECSELs
}

\author{
C. Robin Head ${ }^{1, *}$, Ho-Yin Chan ${ }^{2, *}$, James S. Feehan ${ }^{2}$, David P. Shepherd ${ }^{2}$, Shaif-ul Alam², \\ Anne C. Tropper ${ }^{1}$, Jonathan H. V. Price ${ }^{2}$ and Keith G. Wilcox ${ }^{1}$ \\ ${ }^{1}$ School of Physics and Astronomy, University of Southampton, Southampton SO17 1BJ, UK \\ ${ }^{2}$ Optoelectronics Research Centre, University of Southampton, Southampton SO17 1BJ, UK
}

\begin{abstract}
We present a mode-locked VECSEL emitting 400-fs pulses at a $3 \mathrm{GHz}$ repetition rate at $1040 \mathrm{~nm}$, amplified by a cascaded ytterbium doped fiber amplifier system to an average power of $40 \mathrm{~W}$. The 3-ps duration amplified pulses are recompressed to their original 400-fs duration using a high-throughput transmission grating compressor. The recompressed pulses are used to generate supercontinuum with two different photonic crystal fibers (PCFs); an all-normal dispersion PCF and a PCF with a zero-dispersion wavelength at $1040 \mathrm{~nm}$, creating spectra with $20 \mathrm{~dB}$ bandwidths of $200 \mathrm{~nm}$ with $3.9 \mathrm{~W}$ average power and $280 \mathrm{~nm}$ with $2.5 \mathrm{~W}$ average power respectively.
\end{abstract}

Keywords: Fiber amplifiers, vertical-external-cavity surface-emitting laser (VECSEL), supercontinuum generation, $\mathrm{GHz}$ repetition rate, ytterbium

\section{INTRODUCTION}

Mode-locked (ML) vertical-external-cavity surface-emitting semiconductor lasers (VECSELs) have recently progressed in both pulse duration and average output power. Pulse durations down to 60 fs in pulse groups ${ }^{1}$ and 107 fs for single pulse operation ${ }^{2}$ have been achieved, however with average output powers only on the order of milliwatts. The highest average powers reported to date for sub-picosecond pulses are $5.1 \mathrm{~W}$ with $682 \mathrm{fs}$ pulses ${ }^{3}$ and $5.1 \mathrm{~W}$ with 682 fs pulses. ${ }^{3}$ In continuous wave operation VECSELs have achieved average powers of over $100 \mathrm{~W},{ }^{4}$ but it is not clear yet whether sub $100 \mathrm{fs}$ pulse durations at high average powers will be achieved. A capability unique to VECSELs is the continuous tuning of the repetition rate over several $\mathrm{GHz},{ }^{5,6}$ without drastically changing the average power or pulse duration, due to the mode locking dynamics.

Sub-picosecond pulsed fiber amplifier systems have reached output powers of $830 \mathrm{~W}$ at a $78 \mathrm{MHz}$ repetition rate with a 640 fs pulse duration using a chirped pulse amplification (CPA) architecture. ${ }^{7}$ Due to the nature of CPA systems, however, the amplified pulse has a longer duration than the original seed pulse. As the repetition rate increases, the peak power decreases and the linear amplification regime extends to correspondingly higher average powers before pulse stretching becomes necessary. Recently a fiber master oscillator - power amplifier (MOPA) system has achieved $110 \mathrm{~W}$ at a $1.3 \mathrm{GHz}$ repetition rate with a 890 fs pulse duration requiring only modest pulse stretching. ${ }^{8}$ Using this method it is possible to exploit the parabolic regime to enable pulse compression to durations shorter than the original seed pulse. ${ }^{9}$

Fiber MOPA systems can thus provide a complementary technology to ML-VECSELs to generate femtosecondpulsed high average power $\mathrm{GHz}$-repetition rate laser systems. In previous work Dupriez et al. reported a femtosecond VECSEL seeded MOPA system where average powers of $53 \mathrm{~W}$ were reached. ${ }^{10}$ It was possible to produce pedestal-free 110 fs pulses, but only with a few hundred milliwatts of the average power due to the power handling of the aluminum reflection grating compressor.

Supercontinuum generation has attracted a lot of interest in the past decades for a broad range of applications such as pulse compression, the design of tunable ultrafast femtosecond laser sources and spectroscopy. ${ }^{11}$ Typical

Further author information: C. R. Head: crh206@soton.ac.uk and H.-Y. Chan: hyc1g11@orc.soton.ac.uk 
ML-VECSEL
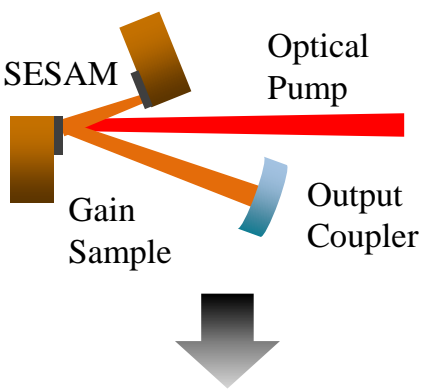

Compressor
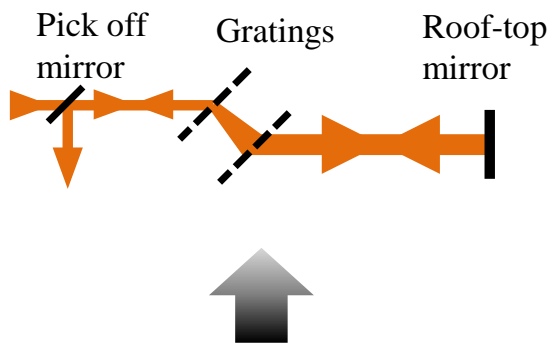

Cascade of Fiber Amplifiers

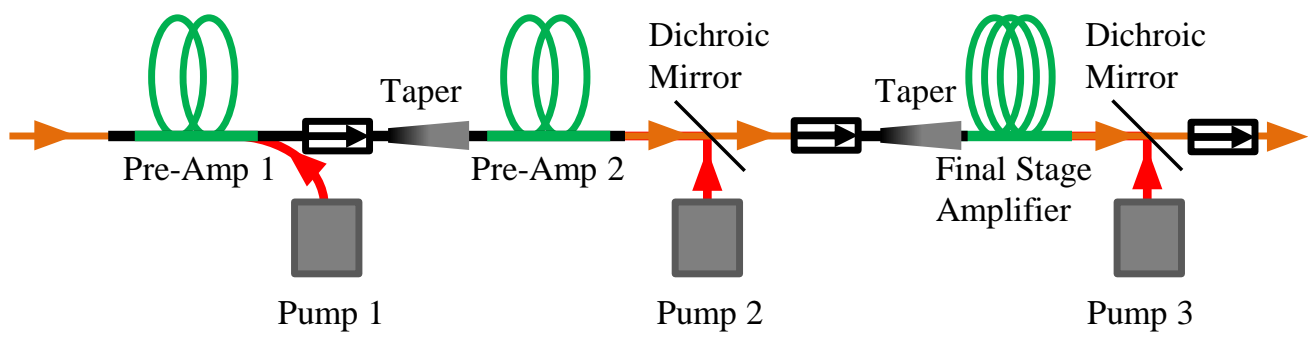

Figure 1. Schematic of the experimental set-up. The VECSEL was used as a seed laser for a three-stage ytterbium-doped fiber amplifier system. The amplified pulses were re-compressed with a high throughput grating compressor and free space launched into the PCF.

laser sources for supercontinuum generation have fixed repetition rates in the kHz-MHz regime. A VECSELMOPA system is of interest as a source of supercontinuum with $\mathrm{GHz}$ repetition rates and has the additional degree of freedom conferred by continuous tuning of the repetition rate.

Chamorovskiy et al. have generated a $650 \mathrm{~nm}$ broad supercontinuum using a 1.57- $\mu \mathrm{m}$ VECSEL-MOPA, producing 15.5 -ps pulses, with a 500 -m long $\mathrm{GeO}_{2}$-codoped silica fiber. ${ }^{12}$

Here we report a VECSEL MOPA system producing $40 \mathrm{~W}$ of average power. The pulses were recompressed to $400 \mathrm{fs}$ and then used to generate supercontinuum in two samples of PCF; an all-normal dispersion PCF, and a PCF with a zero dispersion wavelength of $1040 \mathrm{~nm}$, where spectral widths of $200 \mathrm{~nm}$ and $280 \mathrm{~nm}$ have been achieved, respectively.

\section{EXPERIMENTAL SET-UP}

The VECSEL-MOPA system, shown in Figure 1, consists of distinct sections; The VECSEL, the cascaded fiber amplifier system and the compressor. The compressed output was then launched into the PCF to generate supercontinuum. We describe the VECSEL in section 2.1 followed by the fiber amplifier and pulse compression system in section 2.2 .

\subsection{VECSEL}

The ML-VECSEL in this experiment consists of the gain structure, the semiconductor saturable absorber mirror (SESAM) and a spherical output coupler. The gain structure consists of six strain-balanced InGaAs quantum wells designed for $1025 \mathrm{~nm}$, grown on top of a 27.5 pair AlAs/GaAs distributed Bragg reflector (DBR). The SESAM is used to mode-lock the laser and has a single InGaAs quantum well designed for $1025 \mathrm{~nm}$, on top of 
a DBR designed at $1040 \mathrm{~nm}$. The design and optical properties of the gain structure and SESAM have been described previously by Wilcox et al. ${ }^{13}$ The VECSEL is optically pumped using an $830 \mathrm{~nm}$ fiber coupled diode laser, which is focused to a $60 \mu \mathrm{m}$ radius spot on the gain structure. A V-shaped cavity is formed by a $0.3 \%$ output coupler with a radius of curvature of $50 \mathrm{~mm}$, the gain structure which acts as a plane folding mirror, and the SESAM as the cavity end mirror. The cavity is designed to give a fundamental laser mode with a $60 \mu \mathrm{m}$ radius waist on the gain mirror and a $20 \mu \mathrm{m}$ radius waist on the SESAM, ensuring that the SESAM is saturated more strongly than the gain. The gain structure is mounted on a water-cooled temperature-controlled copper block. Neither the gain nor the SESAM structure has been processed, limiting the maximum average output power. ${ }^{14}$ All components are directly mounted on a single Invar plate to reduce misalignment due to vibrations or thermal drift and thus improve long-term mode-locked stability.

\subsection{Amplifier System}

The MOPA system used in this experiment is similar to the set-up used by Chen et al. ${ }^{15,16}$ but with a VECSEL as master oscillator. The system is based on polarization-maintaining large mode area (LMA) fiber technology, ensuring a robust and stable amplifier system. The first and second pre-amplifiers are ytterbium-doped fibers with core diameters of $5 \mu \mathrm{m}$ and $25 \mu \mathrm{m}$ and lengths of $2 \mathrm{~m}$ and $2.7 \mathrm{~m}$ respectively. Both pre-amplification stages are reverse pumped using $975 \mathrm{~nm}$ fiberized diodes. Following each pre-amplification stage the signal is passed through an isolator. After pre-amplification the signal is free-space-coupled into the final-stage amplifier. The final-stage amplifier fiber, similar to that used in the second pre-amplifier, is a $3 \mathrm{~m}$ length of double-clad LMA ytterbium-doped fiber, which is reverse pumped with a high power $976 \mathrm{~nm}$ fiberized diode laser source (Laserline LDM 200-200). To maintain a single mode beam $\left(\mathrm{M}^{2}<1.1\right)$ the input of the amplifier fiber was pretapered to act as a mode filter as described by Chen et al. ${ }^{15}$ Following the final stage amplifier, the signal passes through an isolator and a high throughput compressor (Ibsen FSTG-PCG-1250-1064 gratings). The amplified and compressed VECSEL signal is free-space coupled into PCF. To improve mechanical durability, and thus allow for a higher average power to be launched, the end-caps of the PCF are collapsed and polished. The two different PCFs used in this experiment are a $10 \mathrm{~m}$ long all-normal dispersion PCF (NKT Photonics NL-1050-NEG-1) with a dispersion minimum at $1050 \mathrm{~nm}$; and a $1 \mathrm{~m}$ long PCF (NKT Photonics SC-5.0-1040) with a ZDW of $1040 \mathrm{~nm}$, closely matched to the wavelength of the amplified signal. 


\section{EXPERIMENTAL RESULTS}

\subsection{Amplifier System}

The target wavelength for the VECSEL seed is $1040 \mathrm{~nm}$ to align approximately with the peak of the gain spectrum of the ytterbium fiber amplifier system. For this particular combination of gain and SESAM wafers, a heatsink temperature of $15^{\circ} \mathrm{C}$ and pump power of $1280 \mathrm{~mW}$ produced a laser spectrum centered at $1040 \mathrm{~nm}$ with a full width half maximum (FWHM) of $2.85 \mathrm{~nm}$, corresponding to near transform-limited 400 fs pulses at a repetition rate of $3 \mathrm{GHz}$ and an average output power of $20 \mathrm{~mW}$. Figure 2 shows the intensity autocorrelation and the optical spectrum of the VECSEL output.
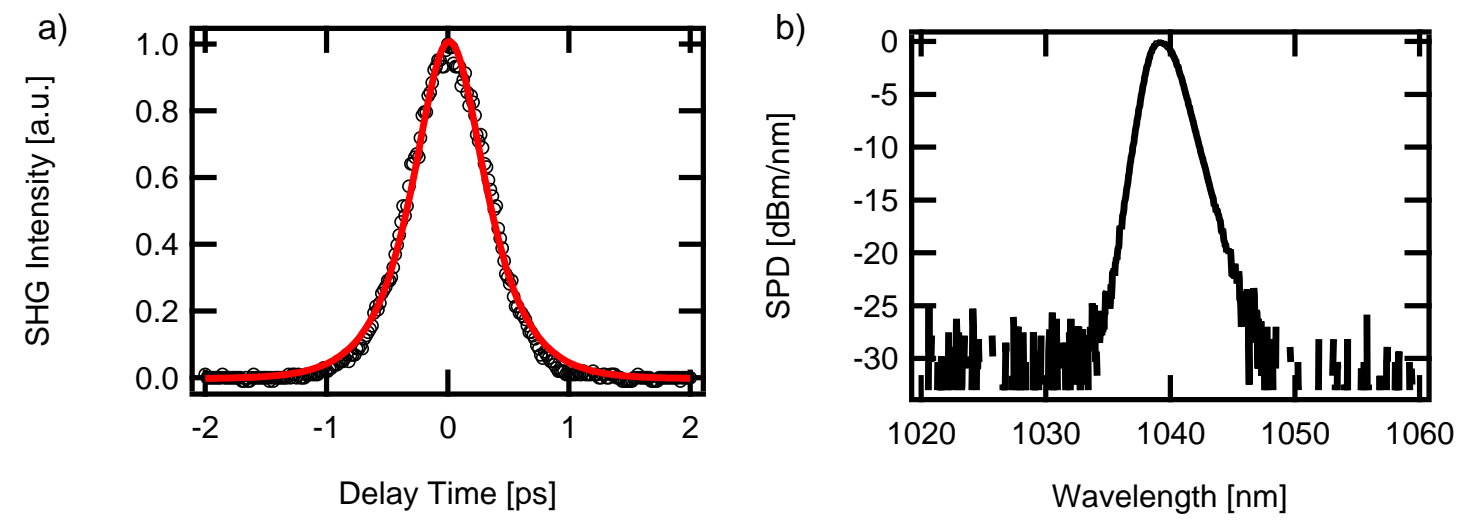

Figure 2. Autocorrelationsand spectrum of the VECSEL. a) Second harmonic generation (SHG) intensity autocorrelation of the pulse (black) with a sech ${ }^{2}$ fit (red). b) The normalized spectral power density (SPD) of the spectrum of the ML-VECSEL. The pulse duration of the VECSEL is $400 \mathrm{fs}$ and the center wavelength of the VECSEL is at $1040 \mathrm{~nm}$ with a full width at half maximum of $2.85 \mathrm{~nm}$.

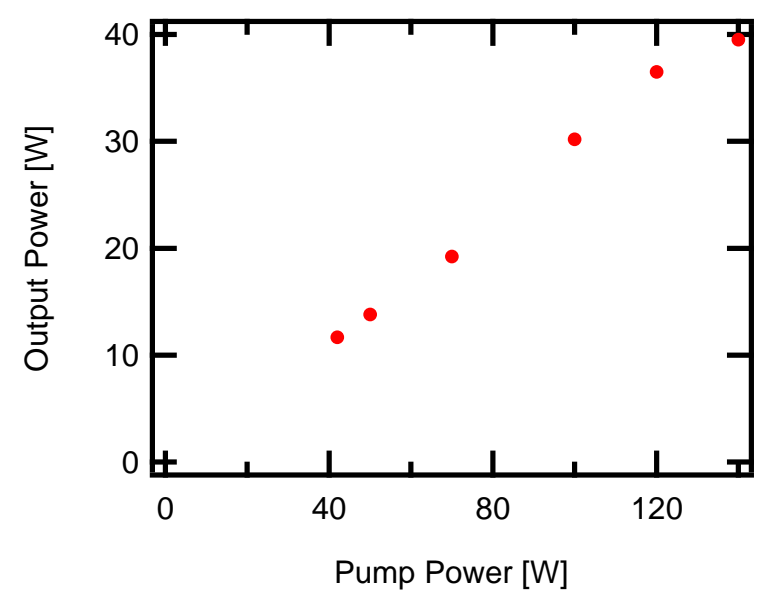

Figure 3. Average power after the amplifier cascade. The maximum average power obtainable was limited by pump absorption in the final stage amplifier fiber.

The average power of the signal after the first two pre-amplification stages was $1.5 \mathrm{~W}$ with a pulse duratios of 1.7 ps. Pump powers of $210 \mathrm{~mW}$ and $5.4 \mathrm{~W}$ were used for the first and second stages respectively. The short-term availability of suitable ytterbium fiber meant that not all of the pump power was absorbed and thus reduced the slope efficiency and compromised the maximum achievable output power in the experiment as seen 

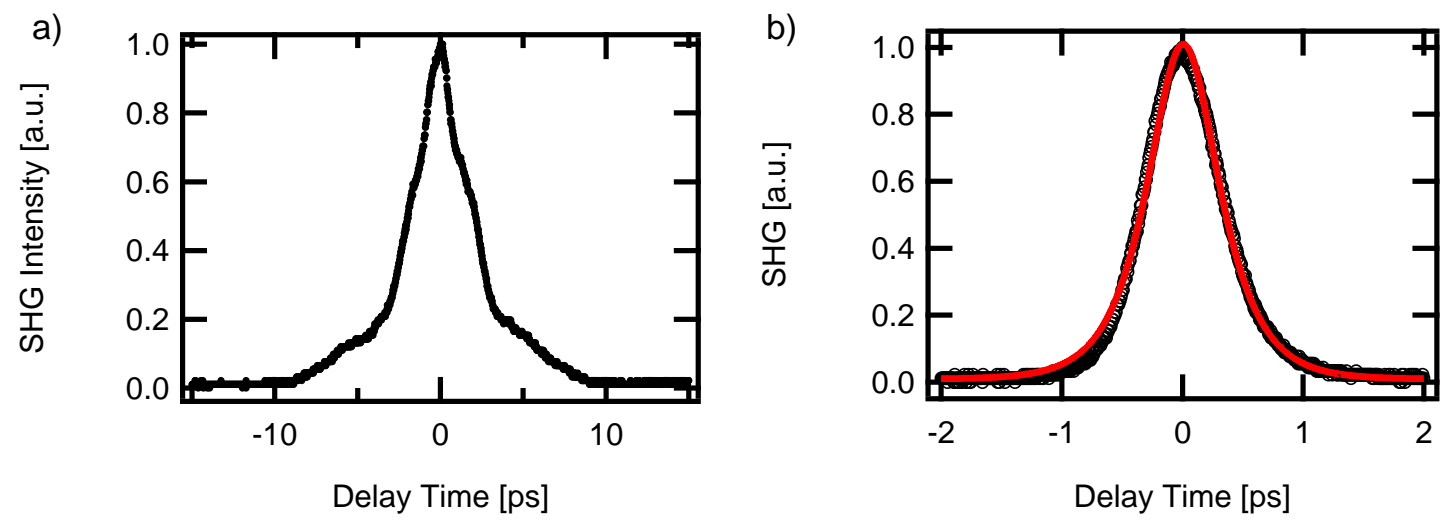

Figure 4. Autocorrelations of the uncompressed and compressed pulse. a) SHG autocorrelation of the uncompressed pulse showing an autocorrelation width of 3 ps. b) The pulses could be cleanly recompressed (black) and were found to have have a 400 fs duration and a $\operatorname{sech}^{2}$ shape (red).

in Figure 3. The pulse duration after the final stage power amplifier was 3 ps at an average power of up to $40 \mathrm{~W}$. The increase in pulse duration was predominantly due to the fiber dispersion in the amplifier system. These average powers were still in the linear amplification regime and so no significant spectral broadening could be observed and no complex phase structure was added by the amplifier chain. Thus it was possible to recompress the pulses to their original duration of $400 \mathrm{fs}$, as shown in Figure 4. Scaling to higher average powers, by using a longer fiber length for the final stage amplifier, should enable parabolic pulse amplification and allow for pulse compression to below 400 fs.

\subsection{Supercontinuum Generation}

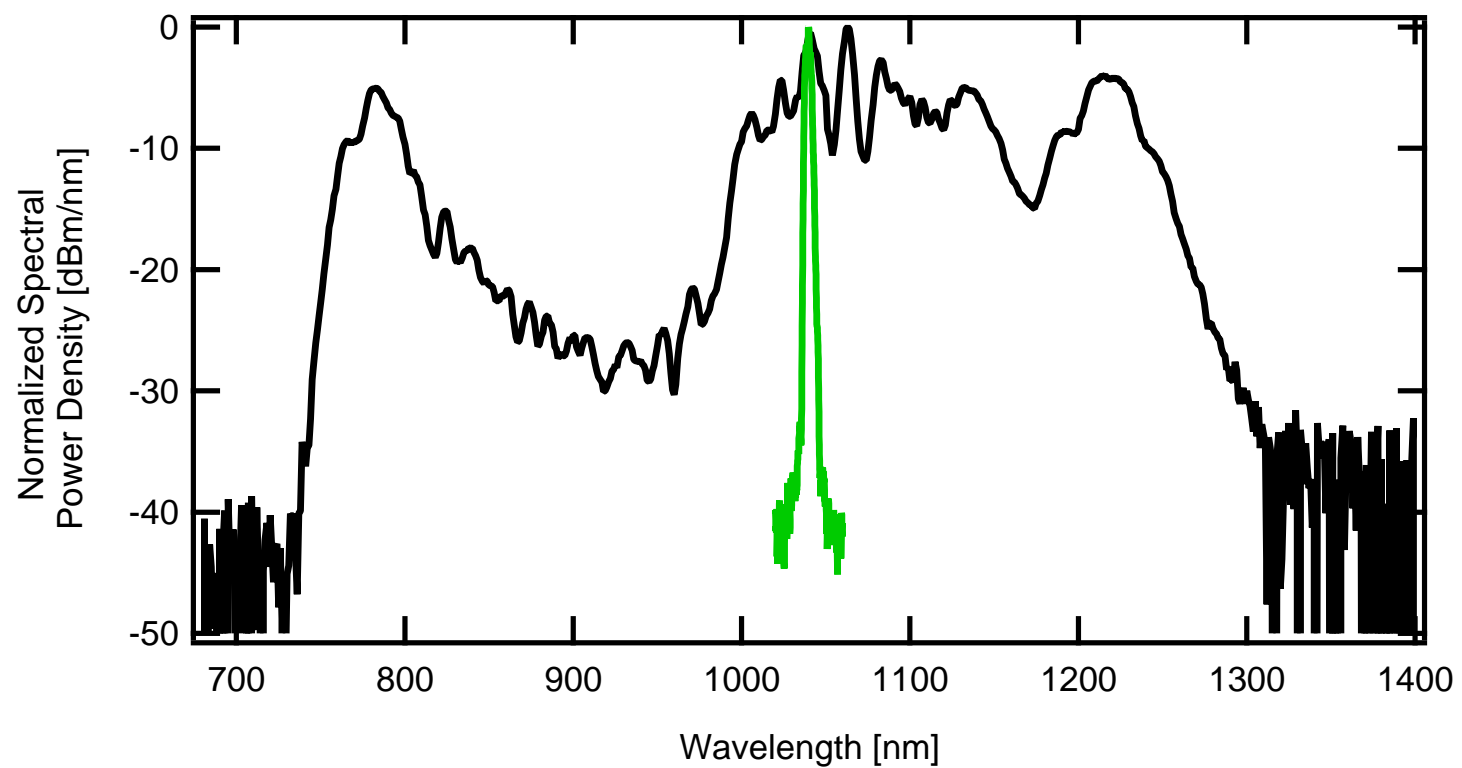

Figure 5. Measured input spectrum (green) and supercontinuum spectrum produced with the PCF with a ZDW of $1040 \mathrm{~nm}$ (black). The $20 \mathrm{~dB}$ bandwidth of the supercontinuum generated is $280 \mathrm{~nm}$

The recompressed 400 fs pulses are launched into the two PCFs in turn, achieving in each case a transmission 


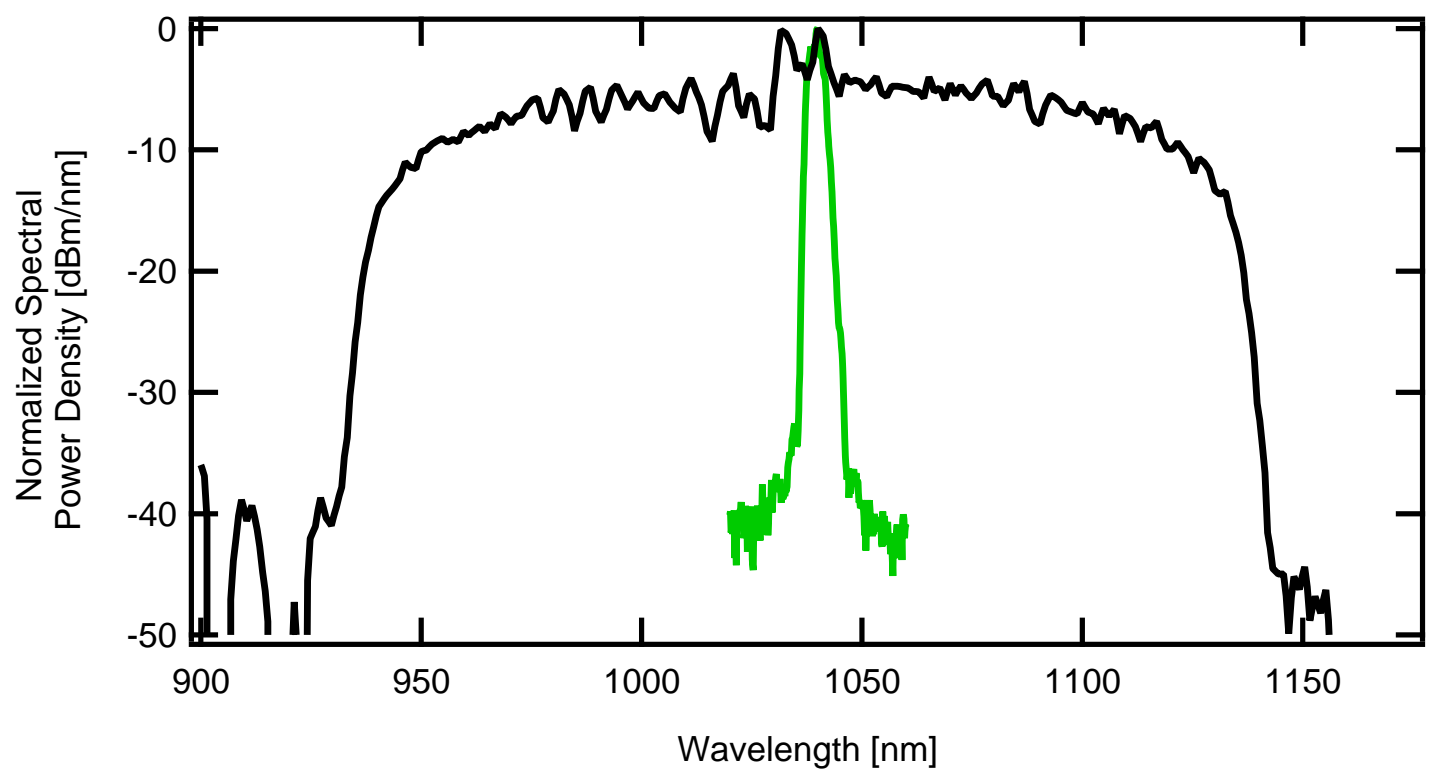

Figure 6. Input spectrum (green) and supercontinuum spectrum produced with the all-normal dispersion PCF (black). The $20 \mathrm{~dB}$ bandwidth of the supercontinuum is $200 \mathrm{~nm}$.

efficiency of $50 \%$ with respect to incident power. The maximum average power measured after the PCF pumped closely to its ZDW was $2.5 \mathrm{~W}$, limited by thermal management of the passively cooled launch into the PCF. Figure 5 shows the supercontinuum spectrum generated with the PCF with a ZDW at $1040 \mathrm{~nm}$. The $20 \mathrm{~dB}$ bandwidth of the supercontinuum created was $280 \mathrm{~nm}$ broad, however the supercontinuum had spectral components between $750 \mathrm{~nm}$ and $1300 \mathrm{~nm}$. This supercontinuum spectrum is mainly driven by soliton dynamics leading to the broader spectrum for this modest peak power at the input, but, it will have low coherence. ${ }^{11}$

In contrast the $20 \mathrm{~dB}$ bandwidth of the supercontinuum created with the all-normal dispersion PCF was $200 \mathrm{~nm}$ as seen in Figure 6. The maximum average power was $3.9 \mathrm{~W}$, again limited by thermal management of the passively cooled launch. The all-normal dispersion fiber produces a broad spectrum through the process of self-phase-modulation and wave-breaking, which require more peak power to obtain a very broad output spectrum, but, since these processes are relatively insensitive to input pulse fluctuations the output has high coherence. ${ }^{17}$

\section{CONCLUSION}

We have demonstrated that the combination of femtosecond ML-VECSELs and ytterbium fiber amplifiers can be used to generate supercontinuum at multi-GHz repetition rates with average powers of several Watts in a robust and practical system. The VECSEL-MOPA produced up to $40 \mathrm{~W}$ output power at a $3 \mathrm{GHz}$ repetition rate where the maximum average power was limited by pump absorption. The amplified pulses could be recompressed to 400 fs durations using a high throughput transmission grating compressor. Supercontinuum with a $20 \mathrm{~dB}$ bandwidth width of $280 \mathrm{~nm}$ and $200 \mathrm{~nm}$ have been generated with PCF pumped closely to its ZDW and with an all normal dispersion PCF, respectively. The average powers of the supercontinuum generated were $2.5 \mathrm{~W}$ and $3.9 \mathrm{~W}$, respectively, limited by the thermally induced input coupling instabilities to the small core $\mathrm{PCF}$, used for supercontinuum generation.

High power supercontinuum generation has been reported by Chen et al. ${ }^{16}$ indicating that with suitably stabilized input coupling and using PCF with optimized dispersion characteristics it should be possible to generate higher average power broader bandwidth supercontinuum in the future. A VECSEL MOPA system employing 
a shorter seed pulse and an optimized final stage amplifier design should enable parabolic regime amplification to produce shorter pulses which could be used to produce octave-spanning supercontinuum.

\section{Acknowledgment}

C. Robin Head and Ho-Yin Chan have contributed equally to this work. We thank R. Malik for his advice and input. This work was undertaken with funding from EPSRC (EP/G059268/1 and EP/I02798X/1). J. H.V. Price was funded by a RAEng/EPSRC Fellowship and K. G. Wilcox holds an EPSRC Early Career Fellowship.

\section{REFERENCES}

[1] A. H. Quarterman, K. G. Wilcox, V. Apostolopoulos, Z. Mihoubi, S. P. Elsemere, I. Farrer, D. A. Ritchie, and A. Tropper, "A passively mode-locked external-cavity semiconductor laser emitting 60-fs pulses," Nature Photonics 3, pp. 729-731, 2009.

[2] P. Klopp, U. Griebner, M. Zorn, and M. Weyers, "Pulse repetition rate up to 92 ghz or pulse duration shorter than 110 fs from a mode-locked semiconductor disk laser," Applied Physical Letters 98, p. 071103, 2011.

[3] M. Scheller, T.-L. Wang, B. Kunert, W. Stolz, S. W. Koch, and J. V. Moloney, "Passively modelocked VECSEL emitting 682 fs pulses with 5.1W of average output power," Electronics Letters 48, pp. 588-589, 2012.

[4] B. Heinen, T.-L. Wang, M. Sparenberg, A. Weber, B. Kunert, J. Hader, S. W. Koch, J. V. Moloney, M. Koch, and S. W., "106 W continuous-wave output power from vertical-external-cavity surface-emitting laser," Electronics Letters 48, pp. 516-517, 2012.

[5] K. G. Wilcox, A. H. Quarterman, H. E. Beere, D. A. Ritchie, and A. C. Tropper, "Variable repetition frequency femtosecond-pulse surface emitting semiconductor laser," Applied Physics Letters 99, p. 131107, 2011.

[6] O. D. Sieber, V. J. Wittwer, M. Mangold, M. Hoffmann, M. Golling, T. Südmeyer, and U. Keller, "Femtosecond VECSEL with tunable multigigahertz repetition rate," Optics Express 19(23), pp. 23538-23543, 2011.

[7] T. Eidam, S. Hanf, E. Seise, T. V. Andersen, T. Gabler, C. Wirth, T. Schreiber, J. Limpert, and A. Tünnermann, "Femtosecond fiber CPA system emitting $830 \mathrm{~W}$ average output power," Optics Letters 35(2), pp. 94-96, 2010.

[8] Z. Zhao, B. M. Dunham, I. Bazarov, and F. W. Wise, "Generation of $110 \mathrm{~W}$ infrared and 65 W green power from a 1.3-GHz sub-picosecond fiber amplifier," Optics Express 20(5), pp. 4850-4855, 2012.

[9] M. E. Fermann, V. I. Kruglov, B. C. thomsen, J. M. Dudley, and J. D. Harvey, "Self-similar propagation and amplification of parabolic pulses in optical fibers," Physical Review Letters 84, pp. 6010-6013, 2000.

[10] P. Dupriez, C. Finot, A. Malinowski, J. K. Sahu, J. Nilsson, D. J. Richardson, K. G. Wilcox, H. D. Foreman, and A. C. Tropper, "High-power, high repetition rate picosecond and femtosecond sources based on Ybdoped fiber amplification of VECSELs," Optics Express 14(21), pp. 9611-9616, 2006.

[11] J. M. Dudley, G. Genty, and S. Coen, "Supercontinuum generation in photonic crystal fiber," Reviews of Modern Physics 78(4), pp. 1135-1184, 2006.

[12] A. Chamorovskiy, J. Kerttula, J. Rautiainen, and O. G. Okhotnikov, "Supercontinuum generation with amplified $1.57 \mu \mathrm{m}$ picosecond semiconductor disk laser," Electronics Letters 48(16), pp. 1010-1012, 2012.

[13] K. G. Wilcox, Z. Mihoubi, G. J. Daniell, S. Elsemere, A. Quarterman, I. Farrer, D. A. Ritchie, and A. Tropper, "Ultrafast optical Stark mode-locked semiconductor laser," Optics Letters 33(23), pp. 2797-2799, 2008.

[14] S. Calvez, J. E. Hastie, A. J. Kemp, N. Laurand, and M. D. Dawson, Thermal management, structure design and integration considerations for vecsels, in Semiconductor Disk Lasers: Physics and Technology, Wiley-VCH Verlag GmbH \& Co. KGaA, Weinheim, Germany, 2010. 
[15] K. K. Chen, J. H. V. Price, S. Alam, J. R. Hayes, D. Lin, A. Malinowski, and D. J. Richardson, "Polarisation maintaining 100W Yb-fiber MOPA producing $\mu \mathrm{J}$ pulses tunable in duration from 1 to 21 ps," Optics Express 18(14), pp. 14385-14394, 2010.

[16] K. K. Chen, J. H. V. Price, S. Alam, J. R. Hayes, D. Lin, A. Malinowski, C. Codemard, D. Ghosh, M. Pal, S. K. Bhadra, and D. J. Richardson, "Picosecond fiber MOPA pumped supercontinuum source with 39 W output power," Optics Express 18(6), pp. 5426-5432, 2010.

[17] A. M. Heidt, "Pulse preserving flat-top supercontinuum generation in all-normal dispersion photonic crystal fibers," J. Opt. Soc. Am B 27(3), pp. 550-559, 2010. 